\title{
Space Charge Properties of PPLP Insulation
}

\author{
Zhiqiang $\mathrm{Xu}$, Wen $\mathrm{Su}$ and George Chen \\ University of Southampton \\ Southampton, United Kingdom \\ Zhq.xu@soton.ac.uk
}

\begin{abstract}
The space charge accumulation in the lapped dielectric has been a concern as it may affect the reliability of the insulation. This study intends to investigate charge dynamics in the polypropylene and in the lapped sample with Kraft paper, with or without the impregnating oil. The space charge profiles were tested using the pulsed electroacoustic technique (PEA) under same DC electric field. Both ionization and charge injection have been found in Kraft paper. For the lapped samples, significant amount of charge has been found accumulated at the interface that has the same polarity as the nearby electrode.
\end{abstract} PEA

Keywords-PPLP; space charge; polypropylene; Kraft paper;

\section{INTRODUCTION}

The lapped type insulation material such as polypropylene laminated paper (PPLP) has been used as a replacement of Kraft paper to improve the AC, impulse and DC breakdown strength and develop lower dielectric loss in some commercial HVDC projects $[1,2]$. PPLP insulation has be used in different type of underground transmission cable such as oil-filled cable, mass-impregnated cable and high temperature superconductor cable $[3,4]$.

Space charge formation in the polymeric cable and charge dynamics in the insulation play a critical role in the efficient and reliable HVDC cable operation. Some works of charge characteristic have been carried out in polymeric insulation, but insufficient in lapped insulation [4]. With its potential use for very high voltage DC cable insulation in perspective, it is important to develop a good understanding of charge dynamics and interface in such laminated insulation. In this paper the laboratory made lapped dielectric samples were studied. Space charge behaviours in different samples were investigated by means of the pulsed electroacoustic (PEA) technique.

\section{EXPERIMENT SETUP}

\section{A. Sample preparation}

To understand the space charge dynamics in the PPLP insulation, both individual part and different combinations of Kraft paper and polypropylene have been tested in this study. Table 1 shows all samples used in this paper.

Polypropylene (PP) film used here is homo-polymer with density of $0.902 \mathrm{mg} / \mathrm{cm}^{2}$ and $100 \mu \mathrm{m}$ thickness. Kraft paper with $80 \mu \mathrm{m}$ thickness was dried in fan oven at $90^{\circ} \mathrm{C}$ for 60 minutes to reduce moisture content. The impregnation oil was Dodecyl Benzene (DDB) oil which has stable aging characteristics. DDB oil was popularly used in MI cable [5]. Two kinds of DDB oil were used for impregnation, undegassed and degassed. The degassed oil was dried in vacuum oven at $60^{\circ} \mathrm{C}$ for 24 hours. The dried samples were impregnated in the DDB oil for 24 hours and the moisture of oil was recorded before and after impregnation. The PP film was laminated by two layers of Kraft paper at $145^{\circ} \mathrm{C}$ under 2.5 tons for 2 minutes and 5 tons for 2 minures. After the lamination, the total sample thickness is about $220 \mu \mathrm{m}$ which is thinner than the sum of the three layers. As the Kraft paper is very easy to absorb moisture when it exposed to air, all the samples were kept in vacuum atmosphere until they were tested.

Table 1 Samples details used in this paper.

\begin{tabular}{|l|c|c|c|}
\hline \multicolumn{2}{|c|}{ Sample } & Structure & Thickness \\
\hline A: PP film & $\begin{array}{c}\text { Impregnated in } \\
\text { undegassed DDB oil }\end{array}$ & $100 \mu \mathrm{m}$ \\
\hline & $\begin{array}{c}\text { Impregnated in } \\
\text { degassed DDB oil }\end{array}$ & $80 \mu \mathrm{m}$ \\
\hline \multirow{3}{*}{$\begin{array}{l}\text { C:PP+Kraft paper } \\
\text { paper }\end{array}$} & PP contact cathode & & $80 \mu \mathrm{m}$ \\
\hline \multirow{4}{*}{ D: PPLP } & PP contact anode & & $170 \mu \mathrm{m}$ \\
\cline { 2 - 4 } & Dried sample & & $220 \mu \mathrm{m}$ \\
\hline & $\begin{array}{c}\text { Impregnated in } \\
\text { undegassed DDB oil }\end{array}$ & & $220 \mu \mathrm{m}$ \\
\cline { 2 - 4 } & $\begin{array}{c}\text { Impregnated in } \\
\text { degassed DDB oil }\end{array}$ & & $220 \mu \mathrm{m}$ \\
\hline
\end{tabular}

B. Experimental eqiupment

The PEA technique has been used to investigate the space charge profile. The PEA system used in this study has a good spatial resolution and sensitivity due to a thin piezoelectric sensor $(9 \mu \mathrm{m}$ thickness) and a narrow width pulse generator (5ns width).

HVDC supply with a positive polarity output was used in this study, the lower electrode is aluminium (cathode) and the upper electrode is semiconducting polymer (anode). The position of both electrodes was marked in the present charge density results. All the experiments were carried out at room temperature and under controlled humidity. Because of the different thicknesses of the samples, the same external electric field was applied to each sample, i.e. $40 \mathrm{kV} / \mathrm{mm}$ in this study. The measurements were taken at various periods during both volts-on (voltage application) and volts-off conditions (temporary removal of the external voltage) and the decay tests were also monitored after the permanent removal of the external voltage.

\section{RESULTS AND DISCUSSION}

\section{A. Charge distribution in SampleA: PP}

The study of charge dynamic in PP film is necessary due to polypropylene is an essential part of PPLP insulation. Sample with $100 \mu \mathrm{m}$ thickness was investigated under $40 \mathrm{kV} / \mathrm{mm}$ and the result of charge distribution is shown in Fig.1. The homo charge injection from both electrodes can be found easily from the Volts-on results. The charge injection takes place very quickly and the charge distribution rarely changes after 2 minutes of voltage application. The charge distribution of Volts-off was measured after removing the applied voltage. 
Obvious negative charge can be found adjacent to the cathode which supports the homo charge injection. The induced positive charges are found at the cathode and the anode as expected. The decay result shows that the injected charge disappears very fast. Most of negative charges near the cathode decay after 30 seconds. It implies that the homo charge injection takes place in at $40 \mathrm{kV} / \mathrm{mm}$ PP film with dominated negative charge and traps in the sample are predominated shallow traps.

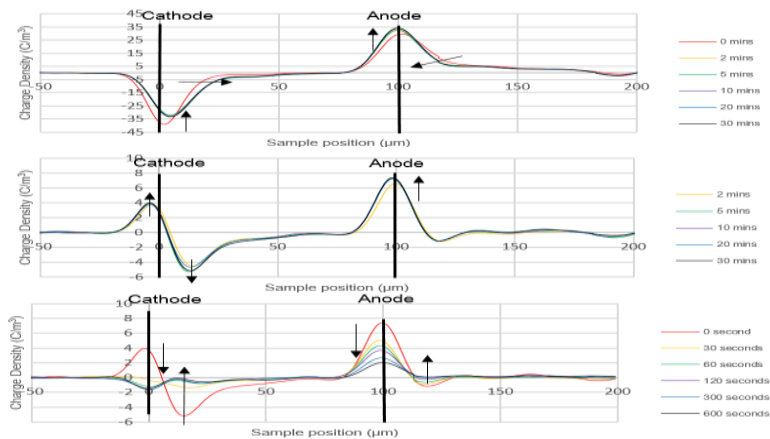

Fig. 1 Space charge distribution in PP film under 40kV/mm applied voltage, top-Volts on, middle-Volts off, bottom-decay.

B. Charge distribution in Sampe B: Kraft paper

The Kraft paper laminated with PP film in PPLP insulation can help the PP overcome the relative incompatibility between film and oil and the problem of insulation oil flowing radially. To understand the charge formation in PPLP it would be helpful if the charge dynamics in paper can be revealed. However, the thin paper without impregnate is prone to breakdown when high voltage is applied. The Kraft paper was impregnated in both undegassed DDB oil and degassed DDB oil respectively. The space charge distribution results of single layer of Kraft paper impregnated in two kinds of DDB oil under $40 \mathrm{kV} / \mathrm{mm}$ are shown in Fig. 2 and Fig.3.

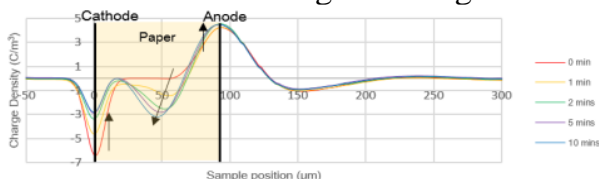

Fig. 2 Space charge dynamics in Kraft paper impregnated by no-degassing DDB oil applied under $40 \mathrm{kV} / \mathrm{mm}$ (volt-on)

The obvious increasing negative peak is found in the bulk of sample close to the anode in Fig.2. It keeps increasing with time following the external voltage application and meanwhile the cathode peak keeps decreasing. For example the cathode peak drops from $6.5 \mathrm{C} / \mathrm{m}^{3}$ to $2.8 \mathrm{C} / \mathrm{m}^{3}$ in 10 minutes. However the anode peak shows a tiny rise.

The decreasing negative cathode peak could be caused by either homo charge injected from the cathode or ionization in the oil-impregnated paper. Thinking about the big negative peak close to the anode, the ionization may be a dominated mechanism in the paper. If it is true, the positive and negative charge will travel to the opposite electrode under the external electric field. The injected negative charge was neutralized by the positive carries in the vicinity of the cathode. Compared with the anode peak the significant decreasing of the cathode peak means negative charge injection from the cathode is stronger than positive injection from the anode in this oilimpregnated Kraft paper.

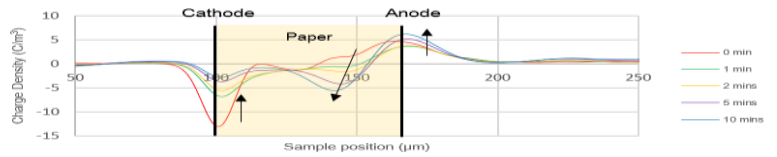

Fig. 3 Space charge dynamics of Kraft paper impregnated by degassing DDB oil applied by $40 \mathrm{kV} / \mathrm{mm}$ (Volt-on)

Fig.3 shows charge dynamics of Kraft paper impregnated in degassed DDB oil. Both ionization and injection occur in the sample based on the similar analyses as above. The negative cathode charge peak decreases and the negative charge in the bulk increases gradually in 10 minutes. On the other hand, positive anode charge peak decreases in first 2 minutes and then increases. At beginning, the charge captured in the area close to the anode is positive (homo charge) then becomes negative (hetero charge) after 10 minutes. And the density of hetero-charge is larger than that of charge accumulated at the cathode. Comparing with Fig.2, the position set apart of the two peaks of negative charge shown in Fig.3 is further away from the cathode. This may imply the both positive and negative homo charge injection are easier and stronger in this degassed oil-impregnated Kraft paper (lower moisture level in oil) than in undegassed oil-impregnated paper (higher moisture level in oil). The neutralization happens much stronger, so the injected negative charges drift deeper into sample than in degassed oil sample.

C. Charge distribution in sample C:PP lapped with Kraft paper

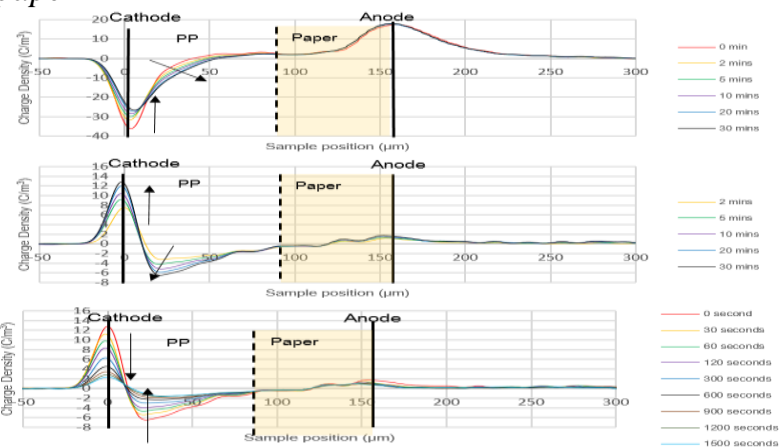

Fig. 4 Space charge distribution of cathode/PP/Kpaper/anode sample under $40 \mathrm{kV} / \mathrm{mm}$ applied voltage, top-Volts on, middle-Volts off, bottom-decay.

In order to have good understand of laminated interface between PP and Kraft paper, one layer PP and one layer of Kraft paper were pressed together. The voltage was applied to Kraft paper side and PP side respectively. The charge distribution results are shown in Fig.4 and Fig. 5. Because of the easy split off the two layer of sample when they impregnated in the oil, dried laminated samples were tested in this section.

The obvious homo charge injection can be found in the sample of PP film in contact with the cathode as shown in Fig.4. From Volts on result, the negative cathode peak decreases gradually over 30 minutes and negative charge move into PP film from the cathode. Meanwhile the anode peak changes very little and there is no obvious charge accumulated 
at the interfaces between PP film and Kraft paper. The reason can be explained as following: The ionization and charge injection occur within the Kraft paper under the external electric field. Both injected positive charge from the anode and negative ions from ionization may exist in the region close to the anode at same time and overlap each other or neutralize. The above processes are difficult to be distinguished by the PEA as the technique only gives the net charge. The positive ions should be accumulated at the interface between PP and Kraft paper, however, no interface peak can be found in the Volts-on and Volts-off results. One of the possible reasons is that the negative injected charges from the cathode are accumulated at or pass through the interface and they neutralize with the positive ions. No peak is found at the interface for the decay results and the negative charges in PP film decay slowly.

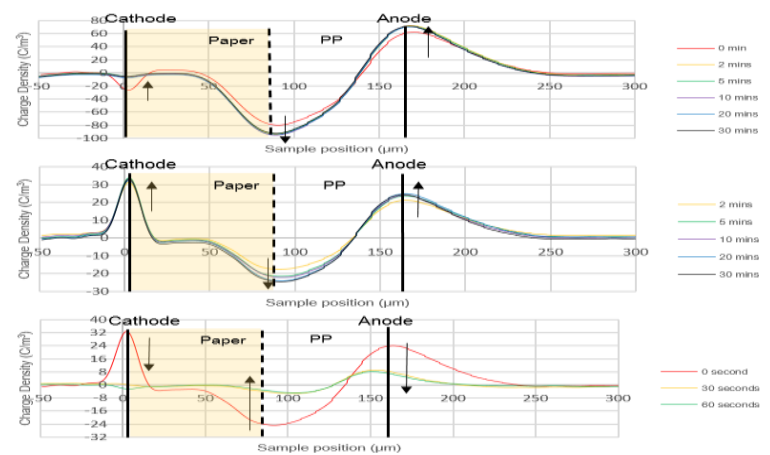

Fig. 5 Space charge distribution of cathode/Kpaper/PP/anode sample under $40 \mathrm{kV}$ applied voltage, top-Volts on, middle-Volts off, bottom-decay.

Fig. 5 shows the result when reversed PP and Kraft paper arrangement. It displays totally different distribution of space charge under the same external electric field. A negative cathode peak can be captured at the moment of applying voltage but drops quickly in 2 minutes. At same time the positive anode peak increases but not obvious moving into bulk of PP layer, which shows a similar pattern to the sample A. A significant negative peak is found at the interface between Kraft paper and PP. The Volts-off results shown in the middle of Fig.5 indicate the negative charge at the interface increases gradually with time and the induced charge at both electrodes. The negative charge at the interface may be the mixture of the injected negative charge from and negative ions from the Kraft paper. The wide shape of the negative peak demonstrates that the significant amount of negative charge is in the bulk of pp adjacent to the interface and the decay result shows that the interface charge drops dramatically quick within the first 30 seconds and a small amount of negative charge left in PP. It is believed that the most of negative charge was released from Kraft paper.

\section{Charge distribution in sample D: PPLP}

PPLP sandwich sample was made with PP between two Kraft paper layers. Three different samples were tested, i.e. dried PPLP sample, impregnated with undegassed DDB oil and degassed DDB oil. The results of these three PPLP samples are shown in Fig.7, Fig.8 and Fig.9.

For dried PPLP, two charge peaks are clearly observed at the interfaces between Kraft paper and PP film, negative on the cathode side and positive on the anode side. The negative peak is found at the cathode and no positive peak at the anode. In the case of the sample $\mathrm{C}$ with Kraft paper to the anode, only a small amount of charge shows at the anode because the injected charge is believed to neutralize with the ions. As the thickness of PPLP sample is larger, the acoustic attenuation and dissipation will be severer, resulting in further reduction for the peak on the anode.

The negative cathode charge decrease with time and the interface peaks increase but the rate of the interface charge increasing is lower than that of the cathode charge decreasing. Some positive charge can be found in the Kraft paper next to the cathode from Volts-on results. It is believed that the both charge injection and ionization happen in both Kraft paper layers in contact with the electrodes. The accumulated charges on both side of PP film (or two interfaces) make the electric field in middle of PP film enhanced. That will help the charge pass the interface barrier and continue to inject into PP film. After removing the applied voltage, the charges accumulated at both interfaces gradually decrease. Almost all the charges disappear after 10 minutes. No charge is left in bulk of PP layer.

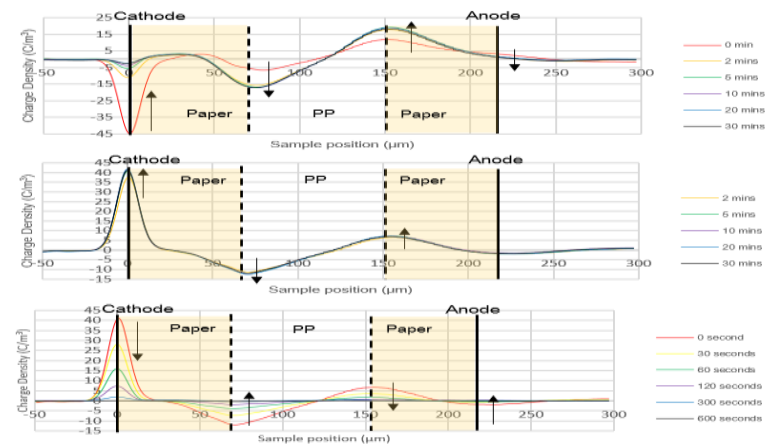

Fig. 6 Space charge distribution of dried PPLP sample under 40kV/mm applied voltage, top-Volts on, middle-Volts off, bottom-decay.

For PPLP impregnated in undegassed DDB oil, shown in Fig.7, the general trend is similar to the dried PPLP results, negative and positive peaks are found from both interfaces between Kraft paper and PP film. However the negative cathode peak is small at the first and decreases quickly to a stable value after 5 minutes. The charge density of the interface peak is much bigger than dried sample. The positive charge in PP film and interface charge gradually increase with time as shown in Volts-off results. After removing the applied voltage, the charges at interface disappear in 30 seconds and charge can only be found at the interface on the cathode side. Negative charge stays in paper side and positive charge is kept in PP film. The interface looks like a new the cathode.

For PPLP impregnated with degassed DDB oil, as shown in Fig.8, similar characteristics are observed within the sample, charge peaks at both interface and positive charge in PP film. However the charge density of interface is much lower, about half value of that in undegassed sample. The negative peak at the cathode drops quickly than the time required for performing measurement. Obvious positive charge is found in the region of Kraft paper close to the cathode. Again, the charge accumulated at interfaces decays 
very fast. The cathode like interface appears and positive charge stays on pp layer side and negative charge on Kraft paper side. This suggests that the positive charge accumulated at the interface on the anode side can move into the middle PP film, resulting in a slow decay.

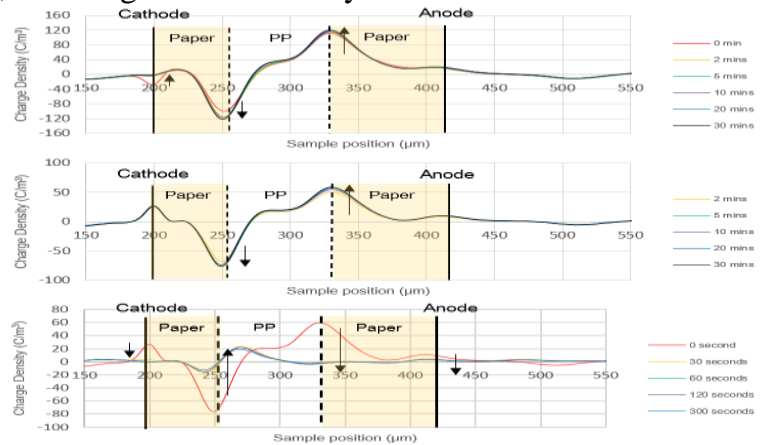

Fig. 7 Space charge distribution of no-degassing DDB oil impregnated PPLP sample under $40 \mathrm{kV} / \mathrm{mm}$ applied voltage, top-Volts on, middle-Volts off, bottom-decay.

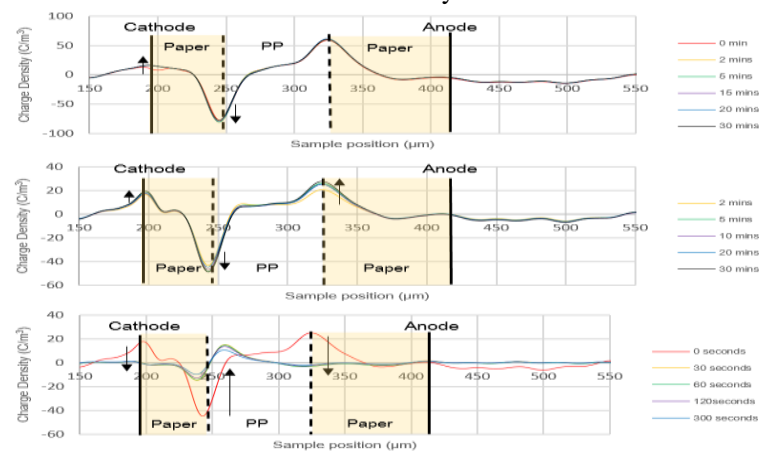

Fig. 8 Space charge distribution of degassing DDB oil impregnated PPLP sample under $40 \mathrm{kV} / \mathrm{mm}$ applied voltage, top-Volts on, middle-Volts off, bottom-decay

\section{DISCUSSTION}

\section{A. Charge in Kraft paper.}

The Kraft paper is made from natural wood pulp and has lots small molecules that can be ionized. It is possible that ionization may take place when the Kraft paper is subjected to the external electric field [6]. Fig. 9 shows a schematic representation of charge formation in PPLP sample under DC electric field. The green charges represent the injected charges and blue charges represent the ions. The charge on the electrode is not drawn in the diagram. The ionization will happen in the Kraft paper when the sufficient high field is applied. Unser the influence of the electric field the positive ions will move in the direction of electric field and negative ions in the opposite direction. For example in the paper next to the cathode, the injected electrons and negative ions in the Kraft paper will move away from the cathode until meet at the interface. The interface serves as a barrier to slow down or trap charge carries. If the interface is between Kraft paper and the anode, the negative charge represented as hetero charge next to the anode, like Fig.2 and Fig.3. If the interface is between Kraft paper and PP film and then anode, a large negative peak will show at the interface, like Fig.5. If the interface is between Kraft paper and PP film and then another Kraft paper, like Figs.6 to 8, negative peak will be definitely found at the interface.

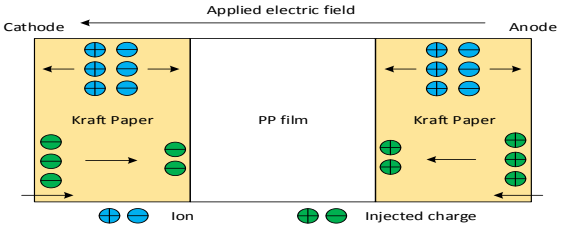

Fig. 9 Illustration of charge in PPLP sample.

The same situation will show in the Kraft paper next to the anode. The positive injected charge and ions will move away from the anode until they meet the interface. When they meet the cathode, the positive charges neutralize with the injected electrons quickly and result in the significant drop of negative cathode peak, like Fig.2 and Fig.3. If they meet the interface with PP and then the cathode on the other side of PP, the positive charge will neutralize with electrons injected from the cathode due to negative injection domination in PP like Fig.4. If the interface with PP then another Kraft paper, like Figs.6 to 8 , positive peak will be found at the interface.

B. The sample moisture content.

Three kinds of PPLP sample were studied for comparison. Dried Kraft paper may absorb the moisture in the process of experiment although the humidity was controlled in the laboratory. Before impregnated, PPLP sample was dried in vacuum. After mixed with dried PPLP, the moisture of undegassed oil decreases from $40.91 \mu \mathrm{g} / \mathrm{ml}$ to $36.48 \mu \mathrm{g} / \mathrm{ml}$. And the moisture of degassed oil increases after mixed with dried PPLP, from $3.75 \mu \mathrm{g} / \mathrm{ml}$ to $17.26 \mu \mathrm{g} / \mathrm{ml}$. There is exchange of moisture between oil and paper, which is dried but still contains moisture. Water itself can be ionised and contribute to the charges formed in the sample. And it can increase the conductivity of materials which affect the speed of charge build-up and movement of charge.

\section{CONLUSIONS}

Space charge behaviors in PP, Kraft paper and laminated dielectric have been investigated under $40 \mathrm{kV} / \mathrm{mm}$ applied electric field in this paper. Fast moving homo charge injection has been found in PP sample. Both injection and ionization charge can be captured in the DDB oil Impregnated Kraft paper. The ionization in the Kraft paper is also a major concern for the lapped sample. Interface in the lapped sample works as a barrier or trap for charge, resulting in charge accumulation at the interface.

\section{REFERENCES}

[1] R. Hata, Y. Yoshino and T. Shimizu, "Application of PPLP to EHV and UHV Class Underground and Submarine Cables, "IEEEPower Engineering Society Winter Meeting, Vol.1, 2000, pp.676-680.

[2] T. Sugata and R. Hata "Development of $500 \mathrm{kV}$ DC PPLP-Insulated Oil-Filled Submarine Cable", CIGRE SC-21 Paris Meeting, 1996

[3] H. Ryosuke, "Solid DC submarine cable insulated with polypropylene laminated paper (PPLP)," SEI Technical Review, p. 3, 2006.

[4] T. Maeno and K. Fukunaga, "Transient phenomena of space charge ditribtutions in polypropylene laminated paper," International Conference on Conduction and Breakdown in Solid Dielectrics, 1998, pp.43-46.

[5] G.Chen, M. Hao, Z. Xu, A.Vaughan, J. Cao and H. Wang "Review of High Voltage Direct Current Cables," CSEE Journal of Power and Energy System, Vol.1, 2015pp.9-21.

[6] C. L. Dawes and P. L. Hoover, "Ionization studies in Paper-Insulated Cable-1" in American Insitute of Electrical Engineers, pp.141-164,1926. 